\title{
Contribution of sVEP visual acuity testing in comparison with subjective visual acuity
}

\author{
Petr Vesely
}

\begin{abstract}
Aims. Visual acuity determination is an important task in ophthalmology and optometry practices. Visual acuity can be examined objectively or subjectively. The objective examination method, SVEP, allows for quick objective measurements of patient's visual acuity. Previous studies have not demonstrated the repeatability of this objective SVEP method. This study aims to evaluate the SVEP method and compare it to a subjective method.

Methods and Results. The sample was divided into two groups. For the first group, visual acuity was measured with SVEP and Snellen methods on only one patient twelve times. In the second group, visual acuity was measured twice with SVEP followed twice with the Snellen method with Landolt's rings and logMAR modification on 32 non-pathological patients. Results showed significant differences between average values of visual acuity obtained with both methods (sVEP and Snellen) in both samples (T-test, $P<0.01$; Wilcoxon test, $P=0.02$ in second group). In the second group, significant correlations between repeated sVEP measurements (Spearman test, $P<0.05, \mathrm{r}=0.69$ ) were found but no significant correlation between average SVEP measurement and average Snellen measurement (Spearman test, $P>$ $0.05, r=0.15$ ) was found.

Conclusion. Objective measurement of visual acuity with SVEP is a valid and reliable method, but is recommended only when it is not possible to use a subjective method for measuring visual acuity, e.g. children, patients with mental retardation or simulating/dissimulating patients.
\end{abstract}

Key words: visual acuity, electrophysiological methods, visual evoked potentials, Snellen chart, ETDRS chart

Received: August 27, 2014; Accepted: January 15, 2015; Available online: February 10, 2015

http://dx.doi.org/10.5507/bp.2015.002

Department of Optometry and Orthoptics, Faculty of Medicine, Masaryk University, Brno and Department of Ophthalmology and Optometry, St. Anne's University Hospital in Brno, Czech Republic

Corresponding author: Petr Vesely, e-mail: veselype@seznam.cz

\section{INTRODUCTION}

Visual acuity testing is one of the important tasks in ophthalmology and optometry practices. Subjective testing of visual acuity depends on the subjective examiner's evaluation and on cooperation from the patient. In the case of preverbal or infantile children, it is better to use an objective method for visual acuity evaluation instead of a subjective method. In these cases, professionals can use an electrophysiological method with visual evoked potential modification for visual acuity determination. The visual system is filtered from the error signal of electrodes, which are attached on a patient's head. Flash or structured stimuli can be used for this method. Rapid recording and evaluation of the signal are a great advantage of the sVEP method (Sweep Visual Evoked Potential). Regan ${ }^{1}$ used this method for evaluation of visual acuity for the first time. Later this method was used for evaluation of visual acuity and contrast sensitivity (Tyler et al. ${ }^{2}$, Seiple et al. ${ }^{3}$, Allen et al..$^{4}$, Norcia et al. ${ }^{5}$ ). Today it is possible to measure two types of visual acuity with the sVEP method - distinguishing (stripes) and coincidence, Vernier visual acuity. For distinguishing visual acuity, stripes with different space frequency are used and the patient's ability to distinguish these stripes is measured. Different space frequency of these stripes is changed during the examination for a period of $10 \mathrm{~s}$ and is defined according to the highest space frequency, which is distinguished by patient. Tyler et al. ${ }^{2}$ showed that sVEP measurements are possible to compare with psychophysical examination of visual acuity, if it is used with proper distinguishing and luminance of testing display.

Before the sVEP method is used, it is very important to set some test parameters which influence measuring results. These include type of stimulus, amount of change of stimulus' space frequency and logarithmic change of space frequency. Gotlobe et al. ${ }^{6}$ recommended logarithmic change of the stimulus, if there is the possibility of covering the visual acuity threshold using the linear change of luminance of stimulus. The advantage of logarithmic change is constant change of stimulus in different visual acuities. Recent studies (Zhou et al. ${ }^{7}$, Bach et al. ${ }^{8}$ ) have shown that the logarithmic distribution of space frequency change for the sVEP method is more suitable. Bach et al. ${ }^{8}$ showed a statistically significant correlation between subjective and objective testing of visual acuity in a group of non-pathological patients, as well as in a group of patients with artificially reduced visual acuity and again in a group of patients with reduced visual acuity for pathological reasons. They used structured field 
and logarithmic change of space stimulus frequency. The authors found the following relationship between subjective and objective visual acuity:

VAs $(\mathrm{dec})=\mathrm{SF} 0 / 17 \mathrm{cpd}($ cycle per degree $)\left(\right.$ ref. $\left.^{1}\right)$

As a testing stimulus, the structured stimulus with stripes oriented in the horizontal or vertical direction, always with changing space frequency, can be used. The direction of space frequency change influences the results. There is the possibility of testing from low frequencies to high or vice versa. Particular space frequencies can be introduced continuously or in samples. Step-introduced stimuli have recently been used.

The sVEP method is also influenced by luminance of testing display. Most professionals use luminance from 40 to $220 \mathrm{~cd} / \mathrm{m}^{2}$. The placement of testing electrodes is also important. sVEP, in comparison with classical VEP method, is not standardised by ISCEV (International Society for Clinical Electrophysiology in Vision). The sVEP method does not have a defined position for active, reference and ground electrodes. Allen et al. ${ }^{9}$ showed that reliable results can be obtained if the active electrode is placed $3 \mathrm{~cm}$ above inion (peak of the occipital bone). Threshold value of visual acuity is obtained from linear extrapolation of $0 \mu \mathrm{V}$ between amplitude VEP value and space frequency (Tyler et al. ${ }^{2}$ ). The main advantage of this method is that variations of particular amplitudes do not influence visual acuity results, but only slope of function (Tyler et al. ${ }^{2}$, Seiple et al. ${ }^{3}$ ). If there is more than one peak of amplitude, the highest peak is used for calculation (Norcia and Tyler ${ }^{11,12}$ ). The Fourier analysis (DFT) for calculation of helping parameters, i.e. signal to noise ratio (SNR) and phase criterion of phase consistence is also used. Norcia et al. ${ }^{12}$ recently recommended testing with SNR ratio larger than 3:1, i.e. amplitude of VEP is three times larger than noise value. Phase consistence criterion means that the visual acuity testing value of phase shift should be nearly the same during all measurements.

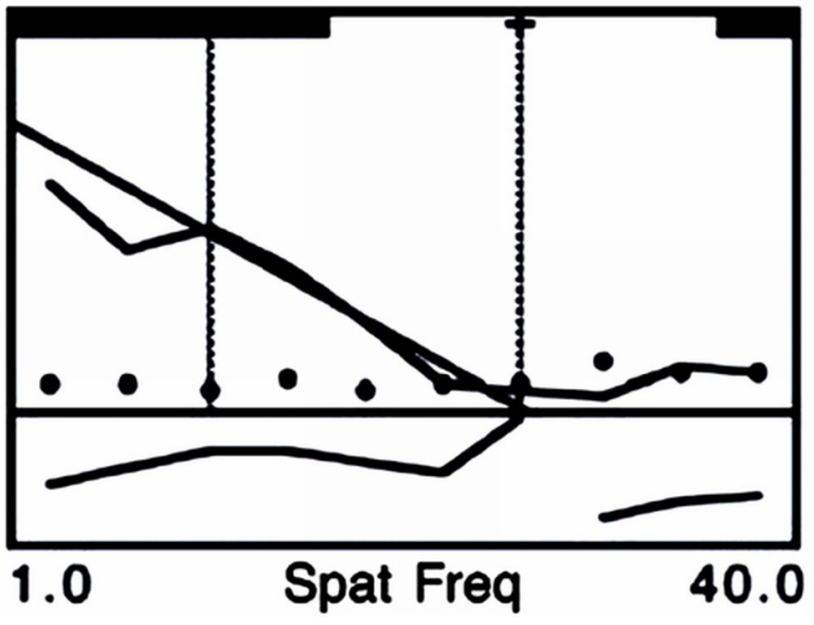

Fig. 1. Calculation of objective visual acuity with extrapolating measured amplitude during sVEP method ( Fahad $\left.^{13}\right)$.

\section{MATERIALS AND METHODS}

The basic sample was divided into two sections. The first sample comprised one man (age 34). His visual acuity was tested repeatedly twelve times in different test times. The second sample comprised 32 subjects (average age 24, min. 21, max. 39). Uncorrected (in emetropes) or spectacle corrected visual acuity with sVEP and Snellen methods were measured on the right eye every time.

The main goal of this study was to evaluate reliability, or the repeatability of sVEP in comparison with Snellen subjective visual acuity testing (group 1). The other goal was to find statistically important differences and correlations between sVEP and the subjective Snellen method in a group of young subjects without eye pathology with or without spectacle correction (group 2).

Visual acuity measuring with sVEP or Snellen method was done in the electrophysiology laboratory in the Department of Eye Diseases and Optometry at St. Anne's University Hospital in Brno. The sVEP method is a modification of the classical Visual Evoked Potentials method (VEP) and is connected to an instrument called Retiscan (Roland Instruments). Examination was done from 1 meter during photopic conditions $\left(80 \mathrm{~cd} / \mathrm{m}^{2}\right)$. The patient wore electrodes according to ISCEV standards for VEP and watches horizontal stripes with changing space frequency. Measuring began on space frequency 2 cycles per degree (cpd) and ended with $25 \mathrm{cpd}$. The changing frequency between black and white stripes was $6 \mathrm{~Hz}$. Contrast of the checker board was set at $60 \%$. The patient wore a black patch on his or her left eye and with right eye watched a red cross in the middle of the screen with structured stimulus. Measuring was repeated with the same method after a 5 min break on the same eye. The study was conducted following the tenets of the Declaration of Helsinki. Informed consent was obtained from all participants.

After $5 \mathrm{~min}$, the subjective visual acuity testing with Snellen chart with Landolt's rings was performed. Visual

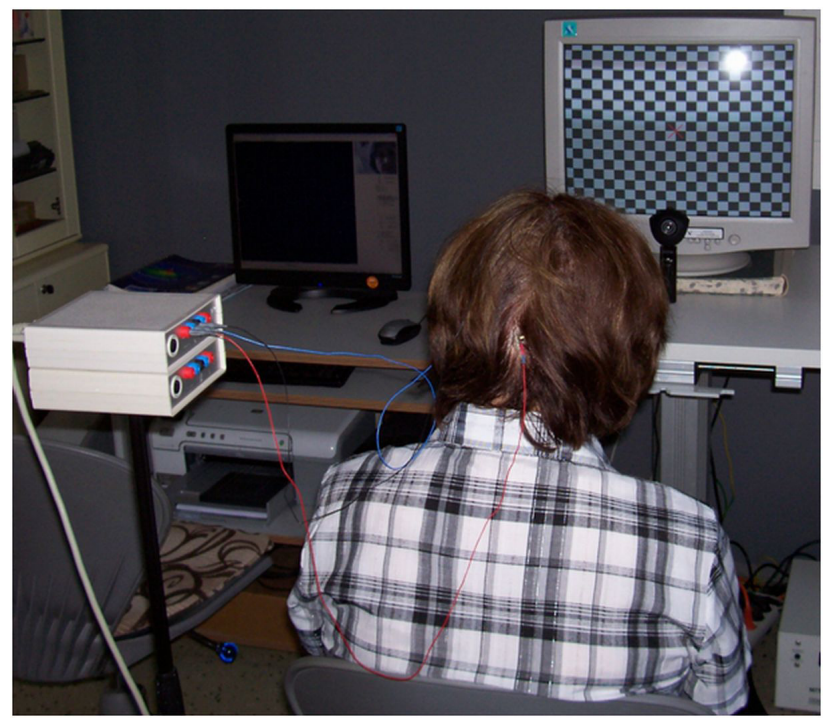

Fig. 2. sVEP visual acuity measuring. 
acuity was noted in $\log$ MAR and converted into decimal notation. After $5 \mathrm{~min}$, this measurement was repeated. We used the programme Statistica (StatSoft) version 12 for statistical evaluation.

\section{RESULTS}

Average value from 12 measurements of visual acuity expressed with cpd using the sVEP method in group 1 was $19.37+/-7.23 \mathrm{cpd}$. The confidence interval (CI, $95 \%$ ) according to Bland and Altman (1986) was $+/-4$ cpd. Average values in the sVEP method expressed logarithmically were $0.23+/-0.19 \log$ MAR and CI was $+/-0.11$ logMAR, or decimally as $0.65+/-0.24$ with $\mathrm{CI}+/-0.14$. In the Snellen method with Landolt's rings, average visual acuity was $0.03+/-0.04 \log$ MAR with $\mathrm{CI}+/-0.02 \log$ MAR, or decimally as $0.94+/-0.09$ with $\mathrm{CI}+/-0.05$. Data in both selection groups for sample 1 was parametrical (Lilliefors test $P>0.02$ ). Hence parametrical testing was used. The correlation coefficient revealed a statistically significant positive correlation between logMAR sVEP and Snellen values $(P<0.05, \mathrm{r}=0.72)$. A t-test showed a statistically significant difference between average sVEP and Snellen values $(P<0.01)$.

In sample 2 for all 32 subjects, the sVEP average value of first measurement was $0.00+/-0.18 \log$ MAR and CI $+/-0.06 \log$ MAR, or decimally as $1.08+/-0.39$ with CI $+/-0.14$ and for the second measurement it was $-0.02+/-$ $0.22 \log$ MAR with $\mathrm{CI}+/-0.08 \log$ MAR, or decimally as $1.21+/-0.78$ with $\mathrm{CI}+/-0.27$. In the Snellen method, aver- age visual acuity values of first measurement were $0.24+/$ $0.45 \log$ MAR with $\mathrm{CI}+/-0.16 \log$ MAR, or decimally as $0.91+/-0.35$ with $\mathrm{CI}+/-0.12$ and in a second measurement was $0.06+/-0.18 \log$ MAR with $+/-0.06 \log$ MAR, or decimally as $0.92+/-0.33$ with $\mathrm{CI}+/-0.11$. The data was non-parametrical as shown by Lilliefors test $(P<0.01)$. Non-parametric tests were used (Spearman and Wilcoxon test). The correlation coefficient showed a statistically significant positive correlation between logMAR sVEP for the first and second measurement values (Spearman test, $P<0.05, \mathrm{r}=0.69$ ), but no correlation between sVEP and Snellen values in logMAR (Spearman test, $P>0.05$, $\mathrm{r}=0.15)$.

Using a Wilcoxon test, the average values of sVEP visual acuity in the first and second measurement period did not significantly differ $(P=0.47)$. Further, the Wilcoxon test showed that the average sVEP and Snellen visual acuity values significantly differed $(P=0.02)$.

\section{DISCUSSION}

Measurement validity for a method is usually obtained by comparing measurements from two different methods and obtaining similar results. Sokol et al. ${ }^{14}$ compared objective measurement of visual acuity from the sVEP technique and preferential looking. His results were from 1.5 to 2.5 octaves ( 1 octave is double or half of cycles per degree) higher with the sVEP method than with preferential looking method. This was probably because the sVEP method determines the threshold value of visual acuity at

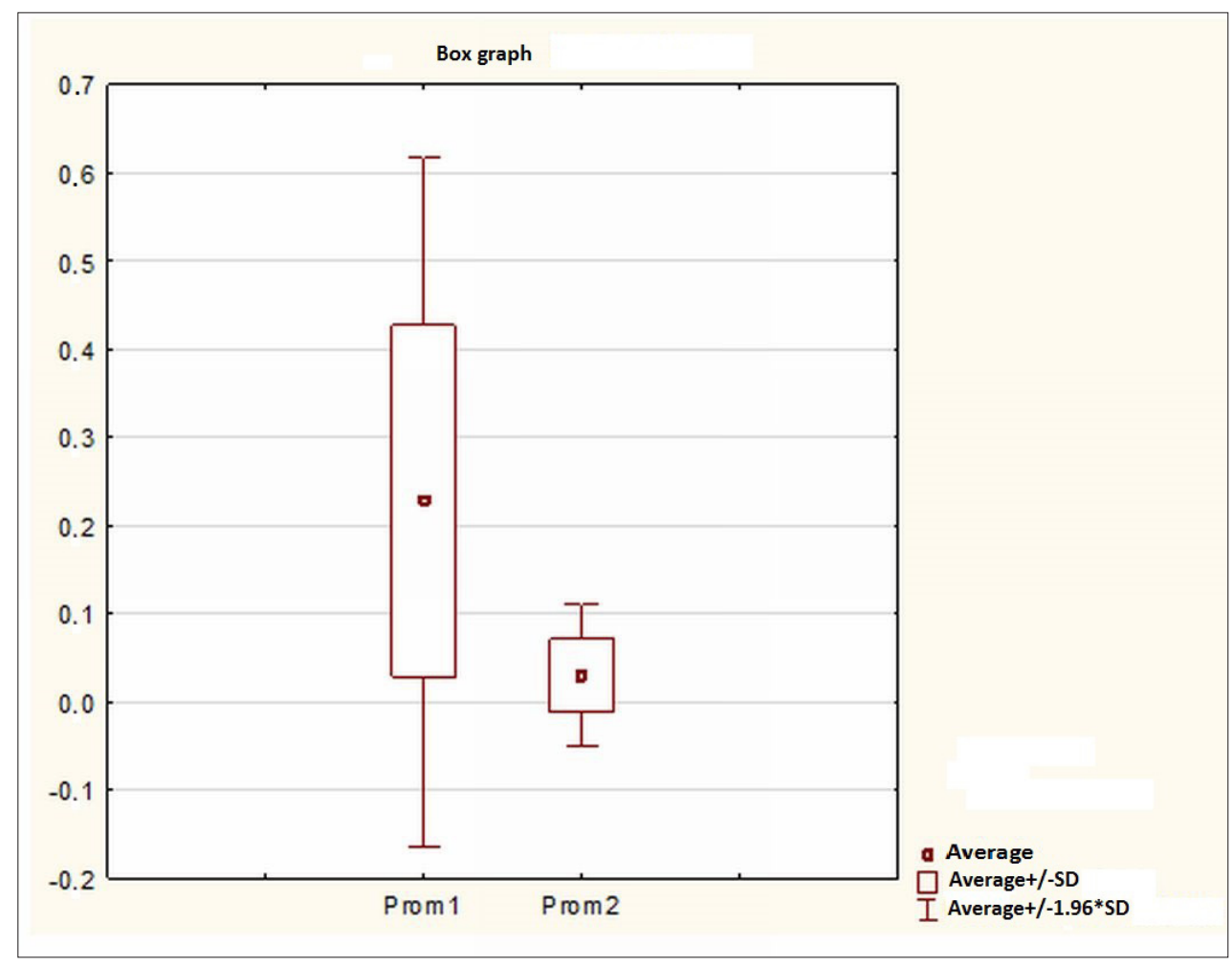

Fig. 3. Statistically significant difference between sVEP (Prom 1, $\log M A R)$ and Snellen (Prom2, logMAR) methods in sample 1. 


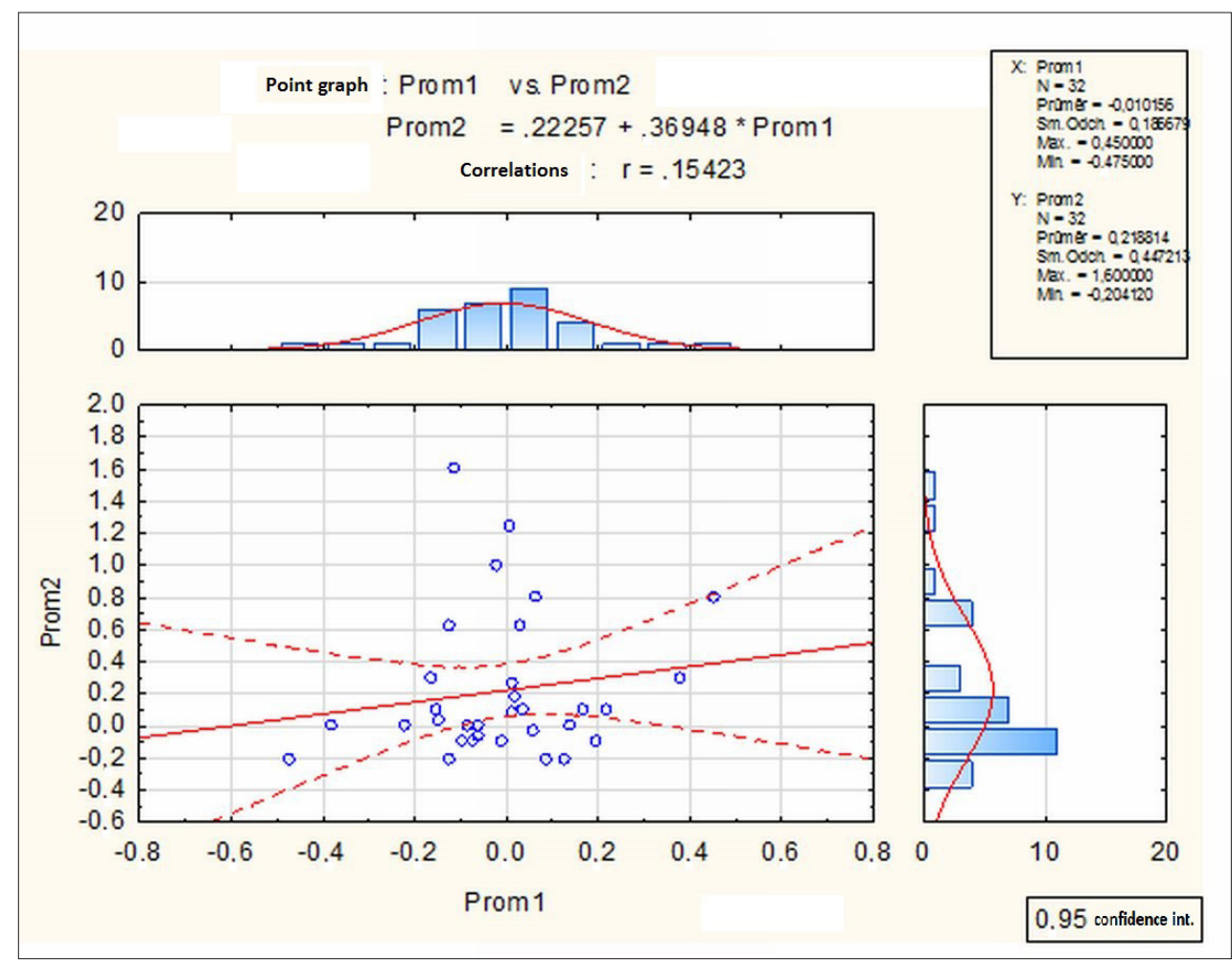

Fig. 4. Statistically no significant correlation $(\mathrm{r}=0.15)$ between average of sVEP (Prom 1$)$ and Snellen (Prom2) values in sample 2 with a significance level $P=0.05$.

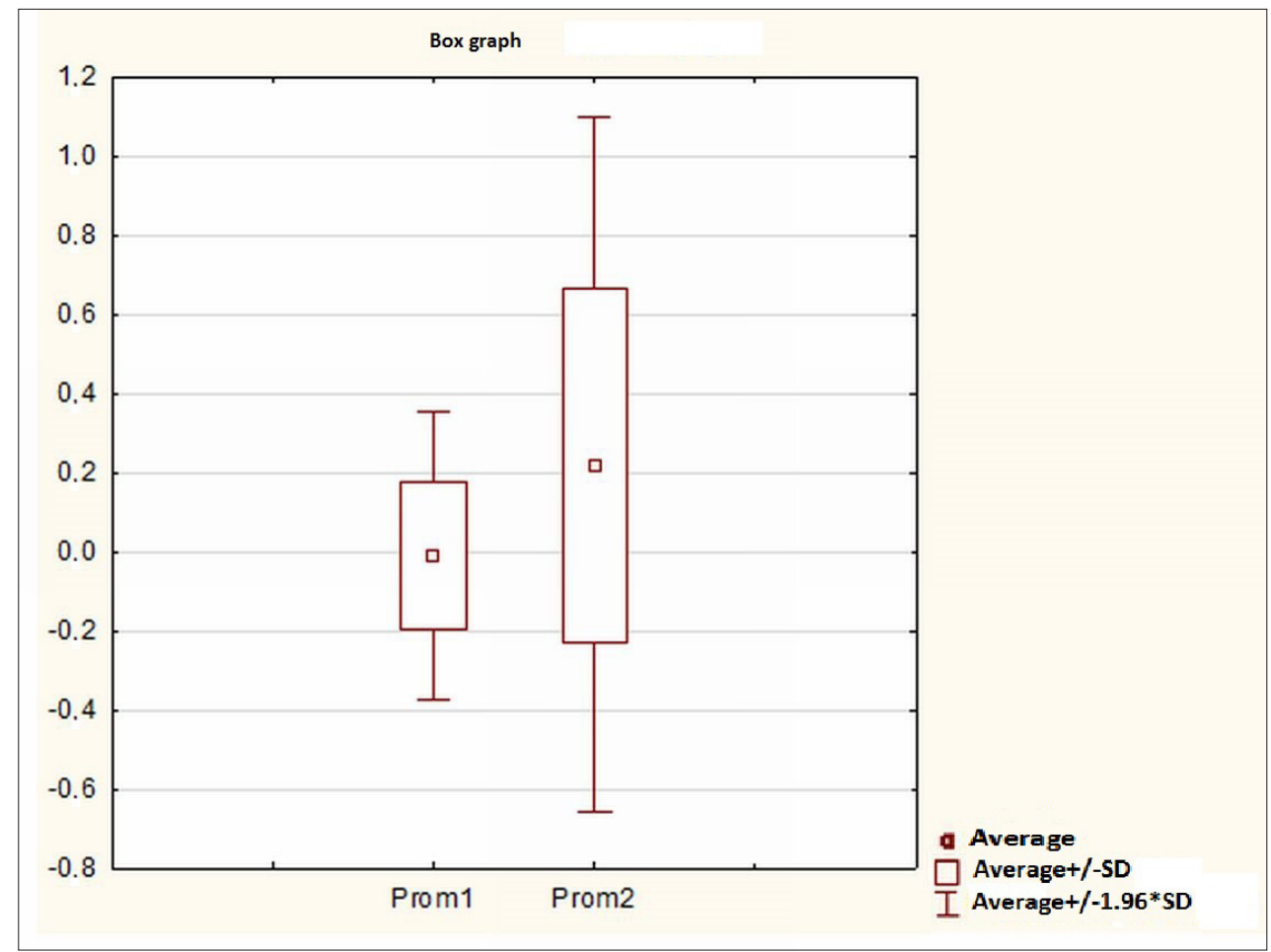

Fig. 5. Statistically significant difference between sVEP (Prom1, logMAR) and Snellen (Prom2, $\log$ MAR) method (Wilcoxon test, $P=0.02$ ) in sample 2 . 
the base of maximal amplitude and the method of preferential looking is based on 70 or $75 \%$ estimation of proper visual acuity threshold. Ridell et al. ${ }^{15}$ showed visual acuity using the sVEP method was higher than with Teller's cards (TAC). Results were shown to deteriorate with age. In another study, Prager et al. ${ }^{16}$ found a lower correlation between visual acuity measured with the sVEP method and Teller's cards $(r=0.3)$. Katsumi et al. ${ }^{17}$ compared visual acuity using the sVEP method with subjective Snellen method in an adult population concluding that measurement of objective visual acuity with black and white stripes was not the same as with Snellen chart. Katsumi et al. ${ }^{17}$ found that visual acuity measured with the Snellen method underestimated in comparison to sVEP group for visual acuity lower than $6 / 18$ and overestimated if visual acuity was higher than 6/30. The authors explained this was due to the limits in LCD monitor resolution in the case of underestimation and due to motion detection and luminescence in the case of overestimation, where stripes are better registered in the sVEP method compared to Snellen. Objective tests are able to prove changes in visual acuity during different visits similarly like subjective tests. But in the case of sVEP, there is a need to set norms for different age groups.

Repeatability of method is measured with repetition of the same method used on different subjects at different times. Norcia and Tyler ${ }^{11}$ measured visual acuity with the sVEP method repeatedly in a group of children and obtained good measurement repeatability - standard deviation (SD) was 0.19 of octave. Hamer et al. ${ }^{18}$ found an average difference lower than 0.25 of octave between test and retest in a group of children older than 10 weeks. Results showed lower differences between test and retest with the use of the sVEP method in comparison to other methods. Prager et al. ${ }^{16}$ found an average difference between test and retest lower than 0.68 of octave. Lauritzen et al. ${ }^{19}$ concluded that variation of visual acuity value based on more than one threshold measurement was more exact than measurement based on best visual acuity measurement. Therefore the sVEP method provided more repeatable results in group visual acuity measurement than in one subject. The current study showed a CI of $+/-0.31 \log$ MAR SD of 0.16 in repeated measurements (first and second) with sVEP in sample 2 and $+/-0.11$ $\log$ MAR in sample 1 .

Since 1980, the sVEP has been used for infant visual acuity measurement. Norcia and Tyler ${ }^{10}$ studied visual acuity development in a group of infants younger than one year. Results showed an increase of visual acuity to 4.5 cpd from first month of life to $20 \mathrm{cpd}$ during $8^{\text {th }}$ and $13^{\text {th }}$ months of life. Adults had visual acuity at $24.3 \mathrm{cpd}$ level. Some authors explained these results due to short stimulus presentation. Hamer et al. ${ }^{18}$ obtained nearly the same values as Norcia and Tyler ${ }^{10}$, i.e. $6 \mathrm{cpd}$ for infants from age 2 to 10 weeks and $14 \mathrm{cpd}$ for infants from age 20 to 30 weeks. Further they studied differences between monocular and binocular visual acuity and found differences maximally 0.2 of octave with an advantage for binocular visual acuity. Binocular visual acuity testing with sVEP has been shown to be more successful than monocular. Norcia et al. ${ }^{12}$ investigated a group of children using sVEP and found that average visual acuity at 2 months of life was from 2.5 to $9 \mathrm{cpd}$ and after 30 weeks ranged from 10 to $20 \mathrm{cpd}$. In a group of adults, values were measured around $31.9 \mathrm{cpd}$. Further they found that in comparison to contrast sensitivity, which reaches the highest point at 10 weeks of life, visual acuity increased after the $30^{\text {th }}$ week of life.

Almost every study mentioned critical value of stripes restoration. Usually $6 \mathrm{~Hz}$ frequency was used, i.e. black and white stripes changed colour more than 12 times. Norica and Tyler $^{10}$ measured visual acuity using a critical frequency from 6 to $10 \mathrm{~Hz}$. Differences in visual acuity were only 0.17 of octave. They did not find statistically significant differences using different critical frequencies ( $10.55 \mathrm{cpd}$ with $6 \mathrm{~Hz}$ and $29 \mathrm{cpd}$ with $10 \mathrm{~Hz}$ ). The present study used a critical frequency of $6 \mathrm{~Hz}$.

The sVEP is a valuable method for measuring visual acuity and also contrast sensitivity in normal healthy subjects and in subjects with eye pathology. The sVEP method is an appropriate technique for visual acuity measurement in patients with spastic cerebral palsy (SCP), because there was underestimation of visual acuity using the subjective method due to motor disability (da Costa et $\left.a{ }^{20}\right)$. Furthermore the sVEP method is preferred in patients with deterioration of visual cortex (CVI), because patients are not able to answer voluntarily regarding visual stimulus $^{21}$. Patients with CVI have problems with holding a target fixation, but they can simply fix a lighted stimulus, so the sVEP method can be used. Alen et al. ${ }^{4}$ and John et al. ${ }^{22}$ described in their studies that by 1 or 2 years of life, subjective visual acuity value rises above the objective value. Arai et al. ${ }^{23}$ compared visual acuity with Snellen and sVEP and found that these methods were very weakly correlated. The lowest correlation was found in a group of patients with neuroretinitis. This premise was also confirmed by Faria et al. ${ }^{24}$ in patients with type 1 diabetes mellitus without retinopathy.

In our study, statistically significant correlations were found between first and second session of sVEP visual acuity measured in sample 2 (Spearman test, $P<0.05$, $\mathrm{r}$ $=0.69$ ). With Wilcoxon test, the average values of visual acuity using sVEP in the first and second session were the same $(P=0.47)$. Repeatability of measurement $(\mathrm{CI})$ expressed in $\log$ MAR was $+/-0.31 \log$ MAR (SD 0.16) in repeated measurements with sVEP in sample 2. This showed that the sVEP method is a valid and reliable method for objective visual acuity measurement. On the other hand, there was a statistical significant difference between Snellen and sVEP measurement in sample 2 (32 measurements) and sample 1 (12 measurements). A Wilcoxon test showed sVEP and Snellen were different in sample 2 $(P=0.02)$ and in sample $1(P<0.01)$. For example, the comparison between sVEP average visual acuity in sample 2 was $-0.01+/-0.20 \log$ MAR versus Snellen $0.15+/-0.32$ $\log$ MAR. Arai ${ }^{23}$ also found a weak correlation between Snellen and sVEP visual acuity measurement $(r=0.66)$. Ridder $^{25}$ in contrast found in a sample of 11 patients with 
normal visual acuity (average $0.00 \log$ MAR) no statistically significant difference between sVEP visual acuity and $\log$ MAR chart (ANOVA, $P=0.16, \mathrm{~F} 20=2.131$ ). Kurtenbach et al. ${ }^{26}$ found $87 \%$ agreement for sVEP in comparison with Freiburg acuity test. Norcia ${ }^{11}$ found in a group of infants younger than 12 months that the sVEP method was more reliable than psychophysical methods (Snellen, logMAR) but was the opposite for a group of adults.

\section{CONCLUSION}

The sVEP method is a very rapid and independent examination and is suitable for infantile visual acuity examination and for some groups of adults. Together with subjective examination, e.g. Snellen chart, sVEP enables better understanding regarding the relationship between visual acuity and contrast sensitivity in a group of infants and adults. In our study, we showed that sVEP is possible for objective visual acuity measurement as no statistically significant differences emerged between sVEP and Snellen in the first and second samples. Thus, the sVEP method is recommended for use only for visual acuity measurement in a group of patients for whom it is not possible to use the subjective method.

\section{ABBREVIATIONS}

sVEP, sweep visual evoked potentials; ISCEV, international society for clinical electrophysiology of vision; $\log$ MAR, logarithm of minimal angle of resolution.

Acknowledgement: This study is supported by a programme of Rector's specific research at Masaryk University Brno (MUNI/A/0886/2012).

I would like to thank the head of Department of Ophthalmology and Optometry Associate Professor Svatopluk Synek M.D., Ph.D. and other colleagues, who cooperated on this project.

Conflict of interest statement: The author state that there are no conflicts of interest regarding the publication of this article.

\section{REFERENCES}

1. Regan D. Rapid objective refraction using evoked brain potentials. Invest Ophthalmol 1973;12:669-79.

2. Tyler CW, Apkarian P, Levi D, K Nakayama. Rapid assessment of visual function; an electronic sweep technique for the pattern visual evoked potential. Invest Ophthalmol Vis Sci 1979;18:703-13.

3. Sieple WH, Kupersmith MJ, Nelson Jl. Evoked potential assessment of cortical adaptation. Appl Opt 1988;27:1089-93.

4. Allen D, Norcia AM and Tyler CW. Comparative study of electrophysi- ological and psychophysical measurement of the contrast sensitivity function in humans. Am J Optom Physiol Opt 1986;63:442-9.

5. Norcia AM, Tyler CW and Allen D. Electrophysiological assessment of contrast sensitivity in human infants. Am J Optom Physiol Opt 1986;61:12-5.

6. Gottlob I, Fendick MG, Guo S. Visual acuity measurement by swept spatial frequency visual-evoked-cortical potentionals (VECPS): clinical application in children with various visual disorders. J Pediatr Ophthalmol Strabismus 1990;27:40-7.

7. Zhou P, Zhao MW, Li XX. A new method for extrapolation th sweep patern visual evoked potential acuity. Doc Ophthalmol 2008:117:8591.

8. Bach M, Maurer JP and Wolf ME. Visual evoked potential-based acuity assessment in normal vision, artificially degraded vision, and in patients. Br J Ophthalmol 2008;92:396-403.

9. Allen D, Bennett PJ, and Banks MS. The effect of luminance of FPL and VEP acuity in human infants. Vision Res 1992;32:2005-12.

10. Norcia AM, Tyler CW. Infant VEP acuity measurements: analysis of individual differences and measurement error. Elecroencephalogr Clin Neurophysiol 1985a;61:359-69.

11. Norcia AM, Tyler CW. Spatial frekvency sweep VEP: visual acuity during the first year of life. Vision Res 1985b;25:1399-408.

12. Norcia AM, Tyler CW and Hamer RD. Measurement of spatial contrast sensitivity with the swept contrast VEP. Vision Res 1989;29:627-37.

13. Fahad A, Susan JL, Irving E. The technique, validity and clinical use of the sweep VEP, Ophthalmol. Physiol Opt 2008;28:393-403.

14. Sokol S, Moskowitz A and McCormack G. Infant grating acuity is temporally tuned. Vision Res 1988;28:1357-66.

15. Riddell PM, Ladenheim B, Mast J. Comparison of measures of visual acuity in infants: teller acuity cars and sweep visual evoked potentials. Optom. Vis Sci 1997;74:702-7.

16. Prager TC, Zou YL, Jensen CL. Evaluation of methods for assessing visual function of infants. J AAPOS 1999;3:275-82.

17. Katsumi O, Arai M, Wajima R. Spatial frequency sweep pattern reversal VER acuity vs Snellen visual acuity: efect of optical defocus. Vision Res 1996;36:903-9.

18. Hamer RD, Norcia AM, Tyler CW. The development of monocular and binocular VEP acuity. Vision Res 1989;29:397-408.

19. Lauritzen $L$, Jorgensen $M H$ and Michaelsen KF. Test-retest reliability of swep visual evoked potential measurements of infant visual acuity and contrast sensitivity. Pediatr Res 2004;55:701-8.

20. da Costa MF, Salomao SR, Berezovsky A. Relationship between vision and motor impairment in children with spastic cerebral palsy: new evidence from electrophysiology. Behav Brain Res 2004;149:145-50.

21. Good WV. Development of a quantitative method to measure vision in children with chronic cortical visual impairment. Trans Am Ophthalmol Soc 2001;99:253-69.

22. John FM, Bromham NR, Woodhouse JM. spatial vision deficits in infants and children with Down syndrome. Invest Ophthalmol Vis Sci 2004;45:1566-72.

23. Arai M, Katsumi O, Paranhos FRL. Comparison of Snellen acuity and objective assessment using the spatial frequency sweep PVER. Graefes Arch Clin Exp Ophthalmol 1997;235:442-7.

24. De Faria JML, Katsumi O, Cagliero E. Neurovisual abnormalities preceding the retinopathy in patients with long-term type 1 diabetes mellitus. Graefes Arch Clin Exp Ophthamol 2001;239:643-8.

25. Ridder WH. Methods of visual acuity determination with the spatial frequency sweep visual evoked potential. Doc Ophthalmol 2004;93:230-47.

26. Kurtenbach A., Buxton W. A comparison of the performance of three visual evoked potential-based methods to estimate visual acuity. Doc Ophthalmol 2013;126:45-56. 